\title{
Adenocarcinoma pulmonar con rearreglo ALK: un fenotipo clínico y tomográfico distinto
}

\author{
ALK-positive lung adenocarcinoma: a different \\ phenotype clinic and tomographic different
}

\author{
Carla Paola Sánchez-Ríos, ${ }^{*}$ Jerónimo Rafael Rodríguez-Cid,* Luis Manuel Martínez-Barrera, ${ }^{*}$ \\ Patricio Santillán-Doherty, Jorge Arturo Alatorre-Alexander*
}

*Instituto Nacional de Enfermedades Respiratorias Ismael Cosío Villegas, Ciudad de México, México.

\begin{abstract}
RESUMEN. Introducción: El tratamiento del cáncer de pulmón actualmente es un modelo que se rige en la selección de pacientes de acuerdo con la presencia de anormalidades oncogénicas. El gen ALK está presente entre 3 y $7 \%$ de los pacientes con cáncer de pulmón de células no pequeñas (CPCNP) y confiere sensibilidad a los inhibidores de ALK. Objetivo: Describir el comportamiento clínico y tomográfico de los pacientes con cáncer de pulmón ALK positivo en el Instituto Nacional de Enfermedades Respiratorias. Material y métodos: Estudio observacional retrospectivo de pacientes con cáncer de pulmón de enero de 2013 a agosto de 2018. Se revisaron expedientes clínicos y radiológicos con registro de variables sociodemográficas, clínicas y moleculares. Resultados: Se analizó una cohorte de 2013 a 2018 con un total de 751 pacientes con diagnóstico de cáncer pulmonar. El CPCNP fue el más frecuente con $93.74 \%(n=704)$. El adenocarcinoma se encontró en $86 \%(n=606)$. Se reportó rearreglo ALK en 3.3\% ( $n=20)$. La mayoría eran de género femenino $65 \%(n=$ 13), la edad promedio fue de $58 \pm 2.4$ años, tabaquismo en $40 \%(n=8)$. De los pacientes nunca fumadores $92 \%(n=11)$ reportaron exposición a biomasa. En 25\% $(n=5)$ se documentó enfermedad pulmonar obstructiva crónica. Los patrones tomográficos fueron: masa-consolidación en 70\% $(n=14)$, engrosamiento pleural con derrame pleural en $20 \%(n=4)$ y patrón micronodular en 10\% $(n=2)$. Conclusiones: Se demuestra un fenotipo clínico y comportamiento diferente al resto de pacientes con CPCNP en pacientes ALK positivo.
\end{abstract}

Palabras clave: Cáncer pulmonar, ALK-rearreglo, pronóstico.

\section{INTRODUCCIÓN}

En los últimos años, el diagnóstico y el tratamiento de pacientes con cáncer de pulmón avanzado han sufrido grandes cambios. El modelo actual para la prescripción de

Correspondencia:

Dra. Carla Paola Sánchez-Ríos

Instituto Nacional de Enfermedades Respiratorias

Ismael Cosío Villegas, Ciudad de México, México.

Correo electrónico: pao1144tost@gmail.com

Trabajo recibido: 06-VIII-2019; aceptado: 03-XII-2019.

\begin{abstract}
Introduction: The treatment of lung cancer currently follows a model that is governed by the selection of patients according to the presence of oncogenic abnormalities. The ALK gene is present in 3 to $7 \%$ of patients with non-small cell lung cancer (NSCLC) and confers sensitivity to ALK inhibitors. Objective: To describe the clinical and tomographic behavior of patients with ALK positive lung cancer in INER. Material and methods: A retrospective observational study of patients with lung cancer from January 2013 to August 2018. A review of clinical and radiological records was made, with registration of sociodemographic, clinical and molecular variables. Results: A cohort was analyzed from 2013 to 2018 with a total of 751 patients diagnosed with lung cancer. The CPCNP was the most frequent with $93.74 \%(\mathrm{n}=704)$. The adenocarcinoma was found in $86 \%(n=606)$. ALK rearrangement was reported in $3.3 \%(n=20)$. The majority were $65 \%$ female $(n=13)$, the average age was $58 \pm 2.4$ years, smoking $40 \%(n=8)$. Of the patients who never smoked, $92 \%(n=11)$ reported exposure to biomass. In $25 \%(n=5)$ COPD was documented. The tomographic patterns were: mass in $70 \%(n=14)$, pleural thickening with pleural effusion in $20 \%(n=4)$ and micronodular pattern in $10 \%(n$ $=2$ ). Conclusions: A clinical phenotype and behavior different to the rest of patients with NSCLC is demonstrated in ALK positive patients.
\end{abstract}

Keywords: Lung cancer, ALK-positive, prognosis.

nuevas terapias especializadas se basa en la selección de pacientes de acuerdo con la presencia de anormalidades oncogénicas específicas en el tumor. ' Las primeras anomalías en ser descubiertas en el cáncer de pulmón fueron las mutaciones en el dominio cinasa del receptor del factor de crecimiento epidérmico (EGFR), seguidas del gen de la cinasa del linfoma anaplásico (ALK), el cual se ha convertido en el segundo oncogén diana en el cáncer de pulmón para el que se han desarrollado nuevas terapias muy efectivas. ${ }^{2}$ Los genes de fusión ALK se identificaron por primera vez en el linfoma anaplásico de células grandes y posteriormente, en el CPCNP y en tumores poco frecuentes como el tumor miofibroblástico inflamatorio. El nuevo gen de fusión ALK 
está formado por un reordenamiento que ocurre en el brazo corto del cromosoma 2 y afecta a los genes que codifican para ALK (2p23.2) y la proteína 4 asociada al microtúbulo del equinodermo (EML4) (2p21) o, rara vez, a los genes en otros cromosomas. El producto proteico de este nuevo gen de fusión tiene una cinasa constitutivamente activa de ALK porque el dominio básico del gen EML4 proporciona un mecanismo para la dimerización de la nueva proteína quimérica. $^{3}$

En general, se han identificado más de 20 patrones de fusión ALK. En cáncer de pulmón, además del patrón principal EML4, se han descrito fusiones con KIF5B, TFG, KLC1 e HIPI. Los puntos de ruptura en el gen ALK casi siempre ocurren en intrón 19 y, rara vez en el exón 20, lo que resulta en una inclusión constante del dominio de quinasa ALK en el gen de fusión/proteína. El EML4-ALK es la fusión de ALK más común encontrada en el CPCNP, se forma por una inversión que ocurre en el brazo corto del cromosoma 2 e involucra los genes que codifican ALK (2p23) y EML4 (2p21), con las variantes 1, 2 y 3a/3b como los patrones de fusión más comunes. Debido a que el reordenamiento del gen implica grandes inversiones cromosómicas y translocaciones, FISH fue el primer método utilizado para detectar todas las formas de reorganización ALK y, hasta hace poco, FISH con sondas de reorganización ALK fueron el criterio de referencia para el diagnóstico de los tipos de cáncer de pulmón con reordenamiento ALK. Más recientemente, la detección de la proteína de fusión ALK por ensayo ALK clona D5F3 por inmunohistoquímica (IHC) recibió la aprobación de la FDA (del inglés, Food and Drug Administration) para la selección de pacientes para ser tratados con un inhibidor de ALK. ${ }^{4}$

El tratamiento con TKI-ALK debe limitarse a los pacientes cuyos tumores contengan esta anomalía. Se prefiere un inhibidor de ALK como terapia inicial para pacientes cuyo tumor contiene esta anomalía genética en países donde está aprobado para esta indicación. Los resultados de un ensayo de fase III que comparó la inhibición de ALK con crizotinib con quimioterapia en pacientes sin tratamiento previo demostraron una prolongación en la supervivencia libre de progresión (SLP) y una mejor tasa de respuesta y calidad de vida. ${ }^{5}$ No se observaron diferencias significativas en la supervivencia general.

Con base en los datos clínicos disponibles, incluido el estudio global ALEX (el Instituto Nacional de Enfermedades Respiratorias Ismael Cosío Villegas [INER], Ciudad de México participó reclutando pacientes mexicanos), se sugiere alectinib para la terapia de primera línea para pacientes con CPCNP positivos para ALK recién diagnosticados. Otras opciones aceptables incluyen ceritinib y brigatinib, aunque entre estos dos agentes brigatinib se prefiere el que se basa en la eficacia sistémica e intracraneal, así como en la tolerabilidad.
El tratamiento con inhibidores de ALK por lo general se continúa hasta que hay evidencia de progresión de la enfermedad. En pacientes cuidadosamente seleccionados (p. ej. un sitio aislado de recurrencia que puede tratarse con terapia local, aquéllos con progresión extremadamente leve y asintomática) se puede continuar con un inhibidor de ALK después de la evidencia inicial de enfermedad progresiva. ${ }^{6}$ En la progresión puede estar indicado el tratamiento con un inhibidor de ALK de próxima generación o con quimioterapia estándar. Para pacientes con ALK positivos que desarrollan resistencia al crizotinib o que no pueden tolerar el crizotinib, recomendamos el tratamiento con uno de los inhibidores de la segunda generación de ALK (brigatinib, alectinib o ceritinib).

El objetivo de este estudio fue describir el comportamiento clínico tomográfico de pacientes con cáncer pulmonar ALK positivo en el INER, así como describir las prevalencias de cáncer pulmonar ALK por género y factor de exposición, además de describir las frecuencias de los diferentes procedimientos diagnósticos y sus complicaciones asociadas.

\section{MATERIAL Y MÉTODOS}

Se trata de un estudio observacional retrospectivo realizado en el Instituto Nacional de Enfermedades Respiratorias Ismael Cosío Villegas de la Ciudad de México, Calzada de Tlalpan 4502, colonia Sección XVI, C.P. 14080, Alcaldía Tlalpan. Se procedió a reclutar expedientes de pacientes con diagnóstico de cáncer de pulmón desde enero de 2013 a agosto de 2018, cuyos datos se determinaron de manera retrospectiva por parte de los investigadores principales para llenado de hojas de cotejo formuladas por los mismos para establecer el perfil clínico y tomográfico donde se determinaron las variables necesarias en relación con los siguientes datos: género, edad, estado de procedencia, sintomatología, toma de muestra, resultado histopatológico, estudios de extensión para estadificación, panel molecular con tinción de IHC. Se evalúo por neumólogo y radiólogo torácico experto en cáncer de pulmón, el perfil tomográfico al diagnóstico de estos pacientes registra el patrón predominante de la neoplasia.

Se incluyeron pacientes que contaban con expediente completo tanto clínico, patológico y tomográfico con positividad documentada para rearreglo ALK por IHC. Se eliminaron aquellos pacientes con expedientes incompletos. Se analizaron los datos mediante estadística descriptiva y los resultados se expresaron en medidas de tendencia central.

\section{RESULTADOS}

Se analizó una cohorte de enero de 2013 a agosto de 2018 con un total de 751 pacientes con diagnóstico de cáncer 
pulmonar en el Instituto Nacional de Enfermedades Respiratorias. Respecto al tipo de cáncer pulmonar, el CNCNP fue el más frecuente con 93.74\% $(n=704)$. De este grupo, la distribución histológica fue: adenocarcinoma en 86\% (n =606) seguido de carcinoma epidermoide $12.6 \%(n=89)$ y células grandes en $1.3 \%(n=9)$. El cáncer de pulmón de células pequeñas representó $6.26 \%(n=47)$ de toda la cohorte. Del grupo de adenocarcinoma pulmonar avanzado $(n=482)$ a los cuales se les envió perfil molecular ante sospecha de ALK, se detectó un total de 20 pacientes con positividad a IHC clona D5F3 en muestra de tejido (4.14\%) (Figura 1).

De los pacientes con rearreglo ALK se observó que 65\% $(n=13)$ eran del género femenino y $35 \%(n=7)$ del masculino. Se registró una edad promedio de $58 \pm 2.4$ años al momento del diagnóstico.

Respecto a los factores de riesgo identificados de cáncer pulmonar, los pacientes con rearreglo ALK de nuestra cohorte reportaron antecedente de tabaquismo en $40 \%$ $(\mathrm{n}=8)$, de los cuales $12 \%$ eran fumadores actuales ( $\mathrm{n}$ $=1$ ), el resto cumplían criterios de exfumadores. De los pacientes nunca fumadores $60 \%(n=12), 92 \%(n=11)$ tenían antecedente de exposición a biomasa; sin embargo, sólo $27 \%(\mathrm{n}=3)$ tenían exposición con índice por arriba de 200 horas al año. Dentro de las comorbilidades respiratorias, sólo $25 \%(n=5)$ tenía diagnóstico funcional de EPOC, en su mayoría por biomasa y del género femenino. El antecedente de exposición a otros factores de riesgo no fue registrado en ningún paciente.

En relación al estado clínico al momento del diagnóstico, $50 \%(\mathrm{n}=10)$ de los pacientes se encontraba con ECOG de 1, 45\% ( $n=9)$ con ECOG de 2 y sólo $5 \%(n=1)$ con ECOG de 3.

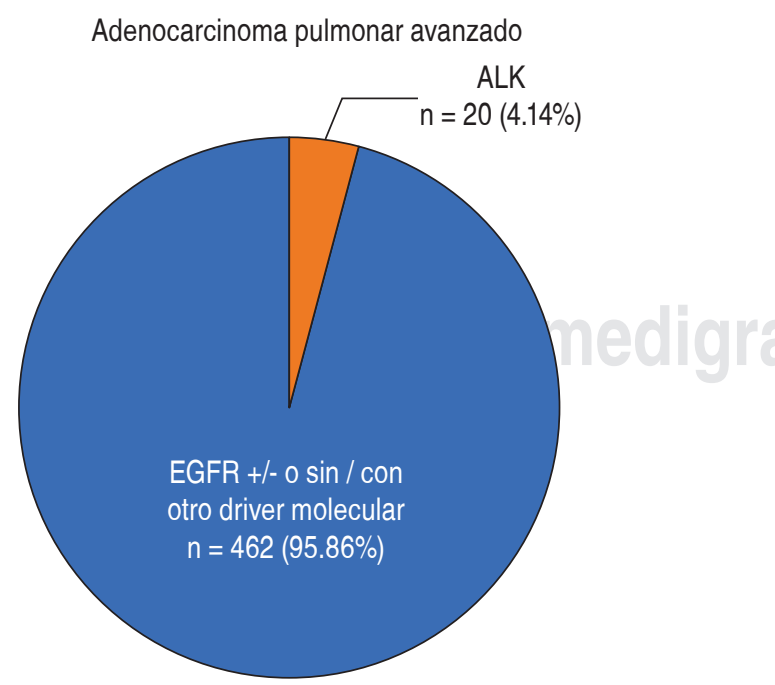

Figura 1: Porcentaje de adenocarcinoma pulmonar avanzado atendido en el INER 2013-2018 con positividad para rearreglo ALK.
Sitios de enfermedad metastásica al diagnóstico

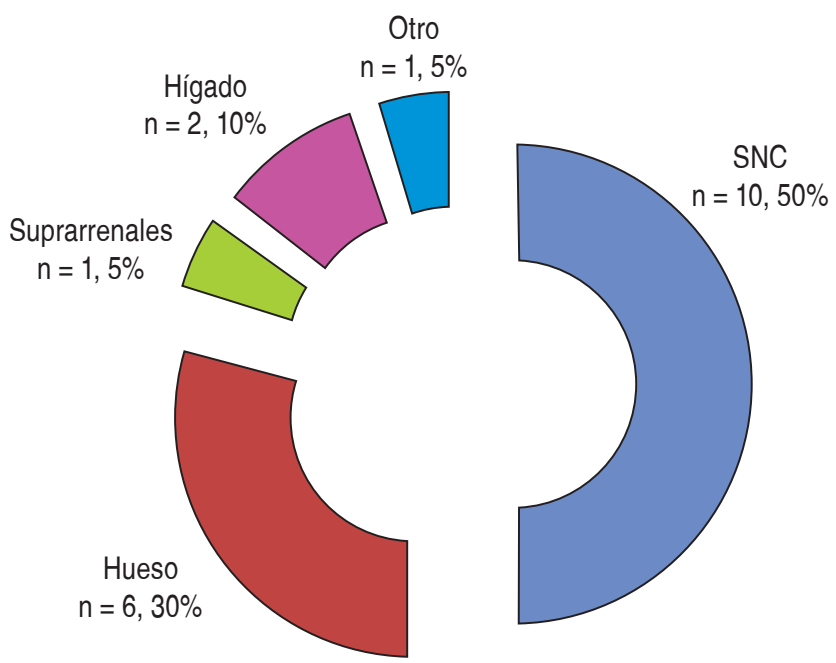

Figura 2: Frecuencia de los sitios metastásicos del adenocarcinoma pulmonar avanzado al momento del diagnóstico.

El estadío clínico al momento del diagnóstico en 100\% $(n=20)$ fue en etapa avanzada. $10 \%(n=2)$ se encontró en etapa IIIB, $60 \%(n=12)$ en etapa IVA y $30 \%(n=6)$ en etapa IVB. Los sitios frecuentes de enfermedad metastásica fueron los siguientes: $50 \%(n=10)$ sistema nervioso central (SNC) y 30\% $(n=6)$ óseo, seguido de $10 \%(n=$ 2) hígado, $5 \%(n=1)$ suprarrenal y $5 \%$ otros $(n=1)$. De los pacientes con enfermedad en SNC 100\% $(n=10)$ se encontró sintomático (Figura 2).

En relación con los patrones tomográficos se observaron como los más frecuentes en orden descendente: patrón de masa en $70 \%(n=14)$, engrosamiento pleural con derrame pleural en $20 \%(n=4)$ y patrón micronodular en $10 \%(n=$ 2). Respecto a la localización de los patrones de masa se observó que $50 \%(n=7)$ en pulmón derecho [LSD 43\%, LM 14\%, LID 43\%], 14.3\% ( $n=2)$ en el pulmón izquierdo [LSI 50\% y LII 50\%], en $21.4 \%(n=3)$ hiliar y en $14.3 \%(n$ = 2) mediastinales (Figura 3).

Respecto a los abordajes diagnósticos se siguieron procedimientos broncoscópicos en $70 \%(n=14)$, de los cuales en $35 \%(n=5)$ se realizó ultrasonido endobronquial. De las biopsias tomadas por fibrobroncoscopia (FBC) $77.7 \%(n=7)$ fueron transbronquiales y $32.3 \%(n=2)$ criobiopsias. Hubo un rendimiento diagnóstico de 100\% con un único procedimiento mínimamente invasivo. Se documentaron complicaciones inmediatas en 14\% $(n=$ 2) por hipoxemia moderada documentada por gasometría arterial (GASA), la cual revirtió a las 48 horas del seguimiento; un paciente con atelectasia y otro con neumonía posobstructiva como hallazgos tomográficos adicionales al patrón principal del tumor. 
Patrones tomográficos de adenocarcinoma pulmonar ALK positivo

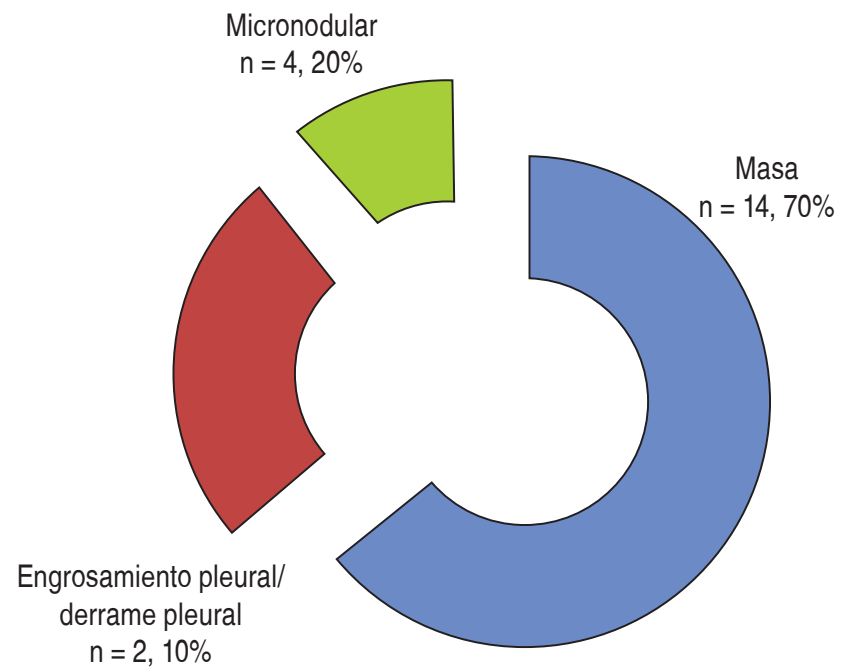

Figura 3: Distribución de los patrones tomográficos del adenocarcinoma pulmonar ALK positivo.

Los procedimientos toracoscópicos se registraron en 20\% $(n=4)$ y los procedimientos guiados por imagen (biopsia pleural cerrada guiada por USG y biopsia transtorácica guiada por tomografía computarizada [TAC]) se realizaron sólo en $10 \%(n=2)$.

Respecto a la información registrada en relación al tratamiento, del total de pacientes, 95\% $(n=19)$ recibió tratamiento de primera línea en el INER y $84.2 \%(n=16)$ recibió esquema a base de quimioterapia. 15.8\% ( $\mathrm{n}=$ 3) pudo recibir tratamiento en primera línea con terapia dirigida (crizotinib) gracias a proyectos de investigación.

\section{DISCUSIÓN}

Tal como se menciona en la literatura mundial y como se refleja en nuestra investigación, la evidencia actual sugiere que el cáncer de pulmón en los nunca fumadores parece ser una enfermedad distinta con presentación tomográfica diferente de aquellos pacientes que desarrollan cáncer pulmonar y tienen como exposición el tabaquismo. Es decir, el CPCNP con rearreglo ALK p. ej. cuenta con una cascada de carcinogénesis y alteración en vías genéticas distintas a las observadas con cáncer de pulmón de fumadores, lo cual les confiere un fenotipo distinto. La edad de presentación de CPCNP con alteraciones genéticas susceptibles de tratamiento es en etapas más tempranas de la vida en comparación con el CP wild type., ${ }^{1,2}$

Se realizó un metaanálisis de datos epidemiológicos para evaluar el perfil de mutaciones de tipos de cáncer de pulmón de pacientes fumadores y nunca fumadores. En esta revisión se identificaron 167 estudios con inclusión de más de 63,000 casos de cáncer de pulmón evaluando razones de probabilidad y se evidenció una probabilidad significativamente mayor de presentar rearreglo ALK en adenocarcinomas en comparación con otras histologías de CPCNP, tal como reveló nuestro estudio. En relación con las alteraciones genéticas, en este metaanálisis se documentó mayor probabilidad que en pacientes nunca fumadores. ${ }^{3}$

Un dato relevante es que la intensidad de exposición al tabaco disminuye de manera proporcional la probabilidad de presentar alteraciones genéticas tipo mutaciones en EGFR, dato que no se ha identificado en rearreglo ALK.4,5

Comparando los CPCNP con mutaciones genéticas tipo ALK y EGFR, los pacientes con rearreglo ALK tienden a mostrar mayor afección a nivel de SNC tanto al momento del diagnóstico como en el sitio de progresión durante la evolución de la enfermedad. En nuestro estudio se documentó afección al SNC al diagnóstico en 50\% de los casos, siendo enfermedad sintomática en 100\% de ellos. Hemos realizado análisis epidemiológicos de nuestros pacientes con mutaciones EGFR y si bien, al igual que el resto de genotipos de cáncer pulmonar se detectan en etapa avanzada, no tienden a presentar tanta afección en el SNC al diagnóstico, siendo en ellos las metástasis óseas el sitio más frecuente de metástasis extratorácicas al momento del diagnóstico. ${ }^{6}$

En relación con los patrones tomográficos de afección, la literatura menciona que el CPCNP con mutación en EGFR no tiene un perfil definido, ya que puede manifestarse con múltiples hallazgos imagenológicos; sin embargo, en comparación con pacientes con CPCNP con rearreglo ALK, tal como reveló el presente trabajo, estos últimos tienden a aparecer como derrames pleurales y masas o áreas de consolidación extensa con broncograma aéreo central que tiende incluso a simular áreas de neumonía de etiología infecciosa, lo que propicia el retardo en la sospecha diagnóstica de cáncer pulmonar en estos pacientes no fumadores.?

\section{CONCLUSIÓN}

Los pacientes con diagnóstico de CPCNP con rearreglo ALK muestran un fenotipo y comportamiento tomográfico distinto al grupo de pacientes que no cuentan con mutaciones oncogénicas detectables susceptibles de tratamiento. En comparación con la quimioterapia estos pacientes tienen mejores respuestas en términos de control de la enfermedad, además se tolera mejor que el perfil de toxicidad de Ios TKI anti-ALK. En nuestra investigación se expusieron las características que hacen diferentes a estos pacientes tal y como se menciona en la literatura mundial.

\section{Agradecimientos}

Limitaciones: estudio descriptivo y retrospectivo, unicentro. 
Neumol Cir Torax. 2020; 79 (1): 26-30

En primer lugar, nuestros resultados son locales, es decir, de un solo centro; sin embargo, consideramos al INER como centro de referencia nacional que concentra gran población de pacientes con factores exposicionales relevantes de cáncer de pulmón diferente al tabaquismo. Segundo, algunos bloques de parafina de pacientes con diagnóstico de adenocarcinoma pulmonar no se enviaron a análisis mutacional por lo escaso de la muestra y la falta de posibilidad de rebiopsias en tejido pulmonar por estado funcional o falta de autorización por parte del paciente. No se dio seguimiento a pacientes a largo plazo en este estudio. Tercero, cabe destacar que los expedientes de pacientes hospitalizados de 2013 a finales de 2017 fueron estadificados con TNM7 que se encontraba vigente y a partir de enero de 2018 se empezó a utilizar TNM8 en el INER para estadificar el cáncer de pulmón. El patrón histológico emitido antes de la clasificación de 2015 de la OMS se reclasificó de manera cegada por el Servicio de Patología. Se determinó IHC ALK con clona D5F3 en tejido en todos los pacientes.

Alcances: Al ser el INER centro de referencia de patologías pulmonares diversas cuenta con un perfil de pacientes susceptibles de desarrollar carcinogénesis pulmonar por otras vías oncogénicas, siendo el grupo de no fumadores del instituto mucho mayor en comparación con otros centros de referencia en riesgo de desarrollar cáncer pulmonar.

\section{REFERENCIAS}

1. Kwak E, Bang YJ, Camidge DR, et al. Anaplastic lymphoma kinase inhibition in non-small-cell lung cancer. N Engl J Med 2010;363(18):1693-1703. doi: 10.1056/NEJMoa1006448

2. Camidge DR, Kono SA, Flacco A, et al. Optimizing the detection of lung cancer patients harboring anaplastic lymphoma kinase (ALK) gene rearrangements potentially suitable for ALK inhibitor treatment. Clin Cancer Res 2010;16(22):5581-5590. doi: 10.1158/1078-0432. CCR-10-0851

3. Chapman AM, Sun KY, Ruestow P, Cowan DM, Madl AK. Lung cancer mutation profile of EGFR, ALK, and KRAS: meta-analysis and comparison of never and ever smokers. Lung Cancer 2016;102:122134. doi: 10.1016/j.lungcan.2016.10.010

4. Tanaka T, Matsuoka M, Sutani A, et al. Frequency of and variables associated with the EGFR mutation and its subtypes. Int J Cancer 2010;126(3):651-655. doi: 10.1002/ijc.24746

5. Shaw AT, Engelman JA. ALK in lung cancer: past, present, and future. J Clin Oncol. 2013;31(8):1105-1111. doi: 10.1200/JCO.2012.44.5353

6. Sánchez-Ríos CP, Rodríguez-Cid JR, Martínez-Barrera LM, SantillánDoherty P, Alatorre-Alexander JA. Descripción clínico-epidemiológica y molecular del cáncer de pulmón en un centro de referencia nacional. Neumol Cir Torax 2019;78(4):356-362. https://dx.doi.org/10.35366/ NT194D

7. Yamamoto S, Korn RL, Oklu R, et al. ALK molecular phenotype in nonsmall cell lung cancer: CT radiogenomic characterization. Radiology 2014;272(2):568-576. doi: 10.1148/radiol.14140789

Conflicto de intereses: Los autores declaran no tener conflicto de intereses. 